\title{
Whole-Course Conformal Radiotherapy Combined with Late-Course Accelerated Hyperfractionation Radiotherapy for Esophageal Squamous Cell Carcinoma: A Report of 110 Cases
}

jianyong sun ( 99785849@qq.com )

Chaozhou City people's Hospital https://orcid.org/0000-0002-2155-928X

weiju huang

Chaozhou City people's Hospital

jingbin chen

Chaozhou City people's Hospital

yaohong zhang

Chaozhou City people's Hospital

\section{Research Article}

Keywords: esophageal carcinoma, conformal radiotherapy, accelerated hyperfractionation, curative effect

Posted Date: April 15th, 2021

DOl: https://doi.org/10.21203/rs.3.rs-411396/v1

License: (c) (i) This work is licensed under a Creative Commons Attribution 4.0 International License.

Read Full License 


\section{Abstract}

Purpose: This retrospective study was designed to analyze the effect and prognostic factors of wholecourse conformal radiotherapy and late-course accelerated hyperfractionation radiotherapy (LCAFRT) for esophageal squamous cell carcinoma (ESCC).

Methods and materials: A total of 110 patients with ESCC received whole-course conformal radiotherapy and LCAFRT in Chaozhou City People's hospital between May 2004 and January 2015. All patients received conventional conformal radiotherapy of $2 \mathrm{~Gy}$ per day up to $30-40 \mathrm{~Gy}$, followed by accelerated hyperfractionation conformal radiotherapy using reduced fields at $1.5 \mathrm{~Gy} /$ fraction twice a day up to 24$39 \mathrm{~Gy}$, with a total dose of 60-69 Gy.

Results: Median follow-up was 85 months (2-170 months). The one-, three-, and five-year survival rates were $81.82 \%, 46.36 \%$, and $41.82 \%$, respectively. The median survival time was 31.8 months. The local control rates for the whole group at 1,3 , and 5 years were $82.73 \%, 70 \%$, and $68.18 \%$, respectively. There were no significant differences among 1-, 3-, and 5-year survival rates and local control rates between the three-dimensional conformal radiotherapy group and intensity-modulated radiotherapy group. The main reactions to acute radiotherapy were acute radiation tracheitis, esophagitis, and pneumonia. Multivariate analysis showed that the tumor location and TNM stage were independent prognostic factors.

Conclusion: The results from this study showed that whole-course conformal radiotherapy and LCAFRT for ESCC can further improve survival and local control with a tolerable acute reaction compared to previous studies. Local recurrence and distant metastasis are the main failure modes of treatment.

\section{Introduction}

Esophageal carcinoma (EC) is one of the most common gastrointestinal cancers in the world. ${ }^{1}$ The incidence rate of EC in different countries or regions is quite different. In North America and Europe the main histological type of EC is adenocarcinoma, while esophageal squamous cell carcinoma (ESCC) is the most common type in Asia, Africa, and South America. ${ }^{2}$ The incidence of EC in China accounts for $50 \%$ of cases around the world, and most of them are ESCCs. ${ }^{3,4}$ There are obvious regional differences in EC in China. High incidence areas are mainly concentrated in the Taihang Mountains. Furthermore, this cancer is particularly prevalent in the Chaoshan area of Southeastern China, with the average agestandardized incidence of about 100/100,000 people. ${ }^{5}$ Radiotherapy is one of the main treatment methods for ESCC, especially for inoperable patients or those unwilling to undergo surgery. In the past, conventional fractionated radiotherapy was the main method of radiotherapy, but its efficacy was limited and the five-year survival rate was at about $10 \%{ }^{6}$ With the development of late-course accelerated hyperfractionation radiotherapy (LCAFRT) for EC, especially in China, its curative effect has been fully confirmed. ${ }^{7,8}$ However, most of the seminal clinical trials have utilized two-dimensional radiotherapy (2DRT) and the number of cases remains small. Since the development of conformal radiotherapy technology in recent years, we conducted a retrospective evaluation of 110 patients with ESCC who 
received whole-course conformal radiotherapy and LCAFRT in order to further verify the efficacy of LCAFRT in ESCC and analyze its treatment failure mode.

\section{Materials And Methods Materials}

The study was approved by our institutional ethics commmittee. Data were collected for 110 patients with EC who received whole-course conformal radiotherapy and LCAFRT in Chaozhou City People's Hospital between August 2004 and January 2015. Pretreatment evaluation of all patients generally included complete history and physical examination, baseline laboratory tests, liver and kidney function tests, fiberoptic esophagoscopy, esophageal barium meal, and chest computed tomography (CT). The eligibility criteria were as follows: (1) initial treatment; (2) age 46-83 years (median age: 65 years) with Karnofsky performance status scores $\geq 70$; (3) all cases were confirmed to be ESCC by pathology; (4) clinical disease stages of I-IVa according to the sixth edition of the TNM staging standard provided by the Union for International Cancer Control (UICC) for esophageal cancer in 2002; and (5) unsuited for chemotherapy or refused chemotherapy. The exclusion criteria included the following: (1) esophageal perforation or deep ulceration; (2) esophageal bleeding; and (3) distant metastases. The results are shown in Table 1. 
Table 1

Patient clinical characteristics

\begin{tabular}{|lll|}
\hline Feature & N & $\%$ \\
\hline No. of patients & 110 & \\
\hline Sex & & \\
\hline Male & 82 & 74.5 \\
\hline Female & 28 & 25.5 \\
\hline Age(years) & & \\
\hline S60 & 51 & 46.4 \\
\hline$>60$ & 59 & 53.6 \\
\hline Median & & 65 \\
\hline Range & & $46-83$ \\
\hline Tumor location & & \\
\hline Cervical & 4 & 3.6 \\
\hline Upper-thoracic & 38 & 34.6 \\
\hline Middle-thoracic & 47 & 42.7 \\
\hline Lower-thoracic & 21 & 19.1 \\
\hline Length (cm) & & \\
\hline$<7$ cm & 70 & 63.6 \\
\hline$\geq 7$ cm & 40 & 36.4 \\
\hline TNM Stage & & \\
\hline I & & \\
\hline II & 48.8 \\
\hline III & 43.6 \\
\hline IVa & 40.0 \\
\hline Radiotherapy mode & & \\
\hline 3 D-CRT & & \\
\hline IMRT & & \\
\hline
\end{tabular}


All patients were treated by external irradiation with 6-mV X-ray linear accelerator. Each patient underwent CT imaging for treatment planning. Then, the images were transferred to a radiotherapy planning system (Eclipse). The target area was delineated by two associate chief physicians, and irradiation field design was based on the diagnosis by CT, esophageal barium meal, and fiberoptic esophagoscopy. The gross tumor volume (GTV) included esophageal primary tumor and regional enlarged lymph nodes. The clinical target volume (CTV) was defined as the GTV expansion of $0.5-0.8 \mathrm{~cm}$ in the radical margin and 2-3 cm in the cranio-caudal margin. The planning target volume is CTV three-dimensional expansion of $0.5 \mathrm{~cm}$. The organs at risk were also sketched, including the spinal cord, lungs, and heart. The radiotherapy plan was designed in 3-5 coplanar fields, including 65 cases of 3D-CRT and 45 cases of IMRT. All patients received conventional conformal radiotherapy of 2 Gy per day up to a dose of 30-40 Gy. It was followed by accelerated hyperfractionation conformal radiotherapy using reduced fields at $1.5 \mathrm{~Gy} /$ fraction twice daily with a minimum interval of 6 hours up to a dose of 24-39 Gy, with a total dose of 60-69 Gy.

End points in this analysis were overall survival, local control, and acute and late radiotherapy responses.

Statistical analysis was performed using SPSS 22.0. Kaplan-Meier survival curves were used to analyze survival and local control rates.

\section{Results}

\section{Follow-up}

As of February 1, 2020, the median follow-up was 85 months (2-170 months), five cases were lost, and the follow-up rate was $93.6 \%$.

\section{Local control and survival rates}

The local control rate for the whole group for 1,3 , and 5 years was $82.73 \%, 70 \%$, and $68.18 \%$, respectively (Fig. 1). The survival rate for the whole group for 1,3 , and 5 years was $81.82 \%, 46.36 \%$, and $41.82 \%$, respectively. The median survival time was 31.8 months (Fig. 2). The 1-, 3-, and 5-year survival rates for 3D-CRT and IMRT groups were $81.54 \%, 44.62 \%, 41.54 \%$ and $82.22 \%, 48.89 \%, 42.22 \%$, respectively. The local control rates were $83.08 \%, 67.69 \%, 64.62 \%$ and $84.44 \%, 73.33 \%, 73.33 \%$, respectively. There were no significant differences (Fig. 3).

\section{Radiation reaction and complications}

The acute toxicities were mainly acute radiation tracheitis, esophagitis, and pneumonia, which could be tolerated without affecting the treatment. The incidence of acute reactions in the IMRT group was lower than that in the 3D-CRT group. The late complications were mainly esophageal stenosis and pulmonary fibrosis (Table 2). 
Table 2

Radiation reaction and complications

\begin{tabular}{|llll|}
\hline Side effects of radiotherapy & N(\%) & 3D-CRT(\%) & IMRT(\%) \\
\hline Acute reactions & & & \\
\hline Bronchitis & $45(40.9)$ & $29(44.6)$ & $16(35.6)$ \\
\hline Esophagitis & $69(62.7)$ & $44(67.7)$ & $25(55.6)$ \\
\hline Pneumonitis & $22(20.0)$ & $15(23.1)$ & $7(15.6)$ \\
\hline Late complications & & & \\
\hline Esophageal stenosis & $8(7.3)$ & $5(7.7)$ & $3(6.7)$ \\
\hline Pulmonary fibrosis & $4(3.6)$ & $3(4.6)$ & $1(2.2)$ \\
\hline
\end{tabular}

Patterns of death and failure

The pattern of death is shown in Table 3. A total of 79 patients died before the follow-up date. The main causes of death were local regional failure and distant metastasis. There were 37 cases of local failure (including distant metastasis) in the whole group, accounting for $46.8 \%$ of the dead cases and including 30 cases of regional recurrence and seven cases of local lesions that were not controlled well. There were 30 cases of distant metastasis (including local failure) in the whole group, accounting for $38.0 \%$ of the dead cases and including 24 cases of distant organ metastasis and six cases of distant lymph node metastasis.

Table 3

Causes of death

\begin{tabular}{|llll|}
\hline Reasons of death & N(\%) & 3D-CRT(\%) & IMRT(\%) \\
\hline Local uncontrolled or & & & \\
\hline regional recurrence & $37(33.6)$ & $25(38.5)$ & 12(26.7) \\
\hline Distant metastasis & $30(27.3)$ & $20(30.8)$ & $10(22.2)$ \\
\hline Hemorrhage & $2(1.8)$ & $1(1.5)$ & $1(2.2)$ \\
\hline Other diseases & 7 & 4 & 3 \\
\hline Unknown & 3 & 2 & 1 \\
\hline
\end{tabular}

\section{Analysis of prognostic factors}

The multivariate analysis results showed that the tumor location and TNM stage were independent prognostic factors (Table 4). 
Table 4

Equation variables

\begin{tabular}{|lllllllll|}
\hline & B & SE & Wald & df & Sig. & $\operatorname{Exp}(B)$ & \multicolumn{2}{c|}{$95.0 \%$ Cl for $\operatorname{Exp}(B)$} \\
\cline { 7 - 9 } & & & & & & & Lower & Upper \\
\hline Sex & .149 & .254 & .345 & 1 & .557 & 1.161 & .706 & 1.909 \\
\hline Age & -.304 & .240 & 1.601 & 1 & .206 & .738 & .461 & 1.181 \\
\hline TNM & -1.531 & .289 & 28.068 & 1 & .000 & .216 & .123 & .381 \\
\hline Lesion location & -.561 & .269 & 4.347 & 1 & .037 & .571 & .337 & .967 \\
\hline Lesion length & -.234 & .240 & .949 & 1 & .330 & .791 & .494 & 1.267 \\
\hline
\end{tabular}

\section{Discussion}

Radiotherapy (RT) is considered to be an effective alternative to surgery for the treatment of patients with EC. ${ }^{9}$ Although the landmark RTOG $85-01$ trial established the CCRT as the standard therapy for EC, its side effects were serious. ${ }^{10}$ Previous studies have found that the efficacy of conventional fractionated radiotherapy is unsatisfactory, the repopulation of surviving tumor cells during treatment with radiation is an important cause of treatment failure, and unconventional fractionated radiotherapy can better inhibit the accelerated proliferation of tumor cells, thus obtaining greater treatment gain ratio. ${ }^{11}$ Based on this concept, LCAFRT for EC has been widely utilized. In the 2DRT era, Shi XH et al. have reported that compared to CFRT, LCAFRT can increase the 5-year survival rate of EC patients from $15-34 \% .{ }^{12}$ Zhao KL et al. have analyzed 201 ESCC patients treated by LCAFRT. Their results have shown that 1-, 3-, and 5-year overall survival rates were $73 \%, 34 \%$, and $26 \%$, respectively, while $1-, 3-$, and 5-year local control rates were $77 \%, 58 \%$, and $56 \%$, respectively. Their treatment resulted in similar local control and survival rates when using standard chemotherapy plus RT, such as was delivered in the RTOG $85-01$ and 94-05. ${ }^{13}$ Several other studies ${ }^{14-16}$ have also indicated that the curative effect of LCAFRT was better than that of CFRT. However, the use of continuous accelerated hyperfractionated radiotherapy ${ }^{17}$ or late-course accelerated hyperfractionation combined with chemotherapy ${ }^{15,18}$ does not significantly increase the efficacy due to toxicity. With the continuous update of radiotherapy equipment in recent years, the conformal radiotherapy technique has been adopted instead of conventional radiotherapy method. Few studies have shown the efficacy of late-course accelerated hyperfractionated conformal radiotherapy. Wang JH et al. have used three-dimensional conformal radiotherapy to treat 98 patients with esophageal cancer, including 48 cases with LCAFRT. The result showed that the 1-, 2-, and 3-year local control and survival rates for LACFRT were $81.3 \%, 62.5 \%$, and $50 \%$ and $79.2 \%, 56.3 \%$, and $43.8 \%$, respectively. However, the radiation side-effects were greater in LACFRT. ${ }^{19}$ Zhang DF et al. have shown that the therapeutic effect of whole-course 3DCRT combined with LCAFRT for EC is superior to conventional radiotherapy, with the 1, 2, and 3-year tumor local control and overall survival rates of $87.8 \%, 75.5 \%$, and $63.3 \%$ and $85.7 \%, 71.4 \%$, and $46.7 \%$, respectively. ${ }^{20}$ In the present study, the five-year local control and survival rates were $68.18 \%$ 
and $41.82 \%$, respectively. Compared to the previous studies, this method offers obvious advantages, which might be due to the fact that conformal radiotherapy elevated the local irradiation dose and homogeneity. The efficacy of 3D-CRT technique or intensity-modulated radiation therapy (IMRT) is similar, but the incidence of radiotherapy-related adverse reactions due to IMRT is lower. The results of multivariate analysis showed that the prognosis of patients with lesions located in the lower thoracic segment and with TNM stage III and IV were poor. In the analysis of the causes of death, local uncontrolled regional recurrence and distant metastasis were still the main causes of death. IMRT may thus be more advantageous for local control.

Overall, the present results revealed that the survival rate for patients with esophageal cancer treated by whole-course conformal radiotherapy and LCAFRT was significantly higher than that for patients treated with conventional technology. IMRT had more advantages in local control and fewer radiotherapy-related adverse reactions. These results need to be further confirmed using prospective studies.

\section{Conclusions}

The results from this study showed that whole-course conformal radiotherapy and LCAFRT for ESCC can further improve survival and local control with a tolerable acute reaction compared to previous studies. Local recurrence and distant metastasis are the main failure modes of treatment.

\section{Abbreviations}

LCAFRT: late-course accelerated hyperfractionation radiotherapy;ESCC:esophageal squamous cell carcinoma;2DRT:two-dimensional radiotherapy;UICC:Union for International Cancer Control;GTV:gross tumor volume;CTV:clinical target volume;IMRT: intensity-modulated radiation therapy.

\section{Declarations}

\section{Ethical Approval and Consent to participate}

The study was approved by our institutional ethics commmittee.

\section{Consent for publication}

Not applicable.

\section{Availability of supporting data}

Not applicable.

\section{Funding}

This study was supported by Chaozhou Science and Technology Bureau (2018GY38). 


\section{Author Contribution}

Study design: SUN Jian-yong and ZHANG Yao-hong. Data acquisition: SUN Jian-yong and CHEN Jing-bin. Statistical analysis and data interpretation: SUN Jian-yong and HUANG Wei-ju.

\section{Conflict of interests}

The authors have no conflict of interests to declare.

\section{Acknowledgement}

We thank International Science Editing (http://www.internationalscienceediting.com) for editing this manuscript.

\section{Authors' information}

Corresponding author:SUN Jian-yong

Address: Oncology Department Chaozhou City People's Hospital, 8 Chengxin Road, 521000 Chaozhou, Guangdong, China

E-mail:99785849@qq.com

Tel: 18666649805 or 13421059885

\section{References}

1. Bray F, Ferlay J, Soerjomataram I, Siegel RL, Torre LA, Jemal A. Global cancer statistics 2018 : GLOBOCAN estimates of incidence and mortality worldwide for 36 cancers in 185 countries. CA Cancer J Clin. 2018;68:394-24.

2. Rustgi AK, EL-Serag HB. Esophageal carcinoma. N Engl J Med. 2014;371(26):2499-509.

3. Chen W, Zheng R, Baade PD, et al. Cancer statistics in China, 2015. CA Cancer J Clin. 2016;66:11532.

4. Lin Y, Totsuka Y, Shan B, et al. Esophageal cancer in high-risk areas of China: research progress and challenges. Ann Epidemiol. 2017;27:215-21.

5. Su M, Liu M, Tian DP, et al. Temporal trends of esophageal cancer during 1995-2004 in Nanao Island, an extremely high-risk area in China. Eur J Epidemiol. 2007;22:43-8.

6. Smalley SR, Gunderson LL, Reddy EK, Williamson S. Radiotherapy alone in esophageal carcinoma: current management and future directions, adjuvant, curative and palliative approaches. Semin Oncol. 1994;21:467-73.

7. Shi XH, Yao W, Liu T. Late course accelerated fractionation in radiotherapy of esophageal carcinoma. Radiother Oncol. 1999;51:21-6. 
8. Zhao KL, Shi XH, Jiang GL, et al. Late course accelerated hyperfractionated radiotherapy plus concurrent chemotherapy for squamous cell carcinoma of the esophagus: a phase III randomized study. Int J Radiation Oncology Biol Phys. 2005;62:1014-20.

9. Sykes AJ, Burt PA, Slevin NJ, Stout R, Marrs JE. Radical radiotherapy for carcinoma of the oesophagus: an effective alternative to surgery. Radiother Oncol. 1998;48:15-21.

10. Cooper JS, Guo MD, Herskovic A, et al. Chemoradiotherapy of locally advanced esophageal cancer:long-termfollow-upof a prospective randomized trial(RTOG85-01). JAMA. 1999;281:1623-7.

11. Kim JJ, Tannock IF. Repopulation of cancer cells during therapy: an important cause of treatment failure. Nat Rev Cancer. 2005;5(7):516-25.

12. Shi XH, Yao WQ, Lui TF. Late course accelerated fractionation in radiotherapy of esophageal carcinoma. Radiother Oncol. 1999;51:21-6.

13. Zhao KL, Shi XH, Guo LJ, Wang Y. Late-course accelerated hyperfractionated radiotherapy for localized esophageal carcinoma. Int J Radiat Oncol Biol Phys. 2004;60(1):123-9.

14. Wang JZ, Fan JJ, Li YY, et al. The efficacy analysis on accelerated hyperfractionaion adiotherapy for esophageal carcinoma. Chin J Radiat Oncol. 2004;13:157-8.

15. Li XM, Zhang RF, Guo D, et al. Clinical analysis for effect of late-course accelerated hyperfractionation for esophageal carcinoma. Chin J Clin Oncol. 2006;33:1025-31.

16. Wang MM, Huang J, Lou YF. Clinical study of late course accelerated hyperfractionation radiation in esophageal carcinoma. Chin J Radiat Oncol. 2007;16:73-4.

17. Wang Y, Shi XH, He SQ, et al. Comparison between continuous accelerated hyperfractionated and late-course accelerated hyperfractionated radiotherapy for esophageal carcinoma. Int J Radiat Oncol Biol Phys. 2002;54:131-6.

18. Zhao KL, Shi XH, Jiang GL, et al. Late course accelerated hyperfractionated radiotherapy plus concurrent chemotherapy for squamous cell carcinoma of the esophagus: a phase III randomized study. Int J Radiation Oncology Biol Phys. 2005;62:1014-20.

19. Wang JH, Lu XJ, Zhou J, Wang F. A randomized controlled trial of conventional fraction and late course accelerated hyperfractionated three-dimensional conformal radiotherapy for esophageal cancer. Cell Biochem Biophys. 2012;62:107-12.

20. Zhang DF, Zhang PL, Wang YG, et al. Evaluation of whole-course three-dimensional conformal radiotherapy combined with late-course accelerated hyperfractionated radiotherapy for esophageal carcinoma patients. Chin J Cancer Prev Treat. 2010;17(7):521-2.

\section{Figures}




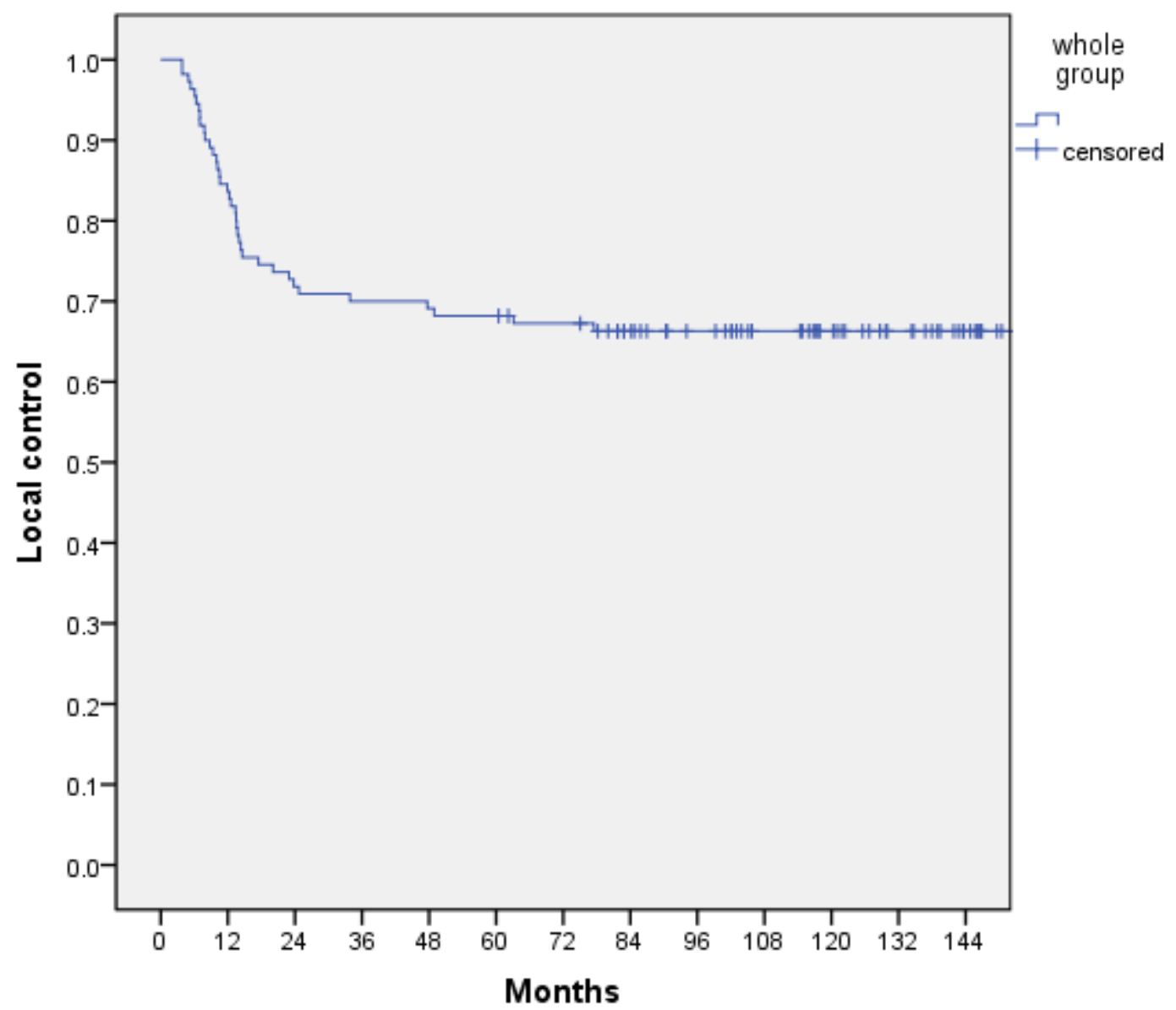

Figure 1

Local control rate for the whole group. 


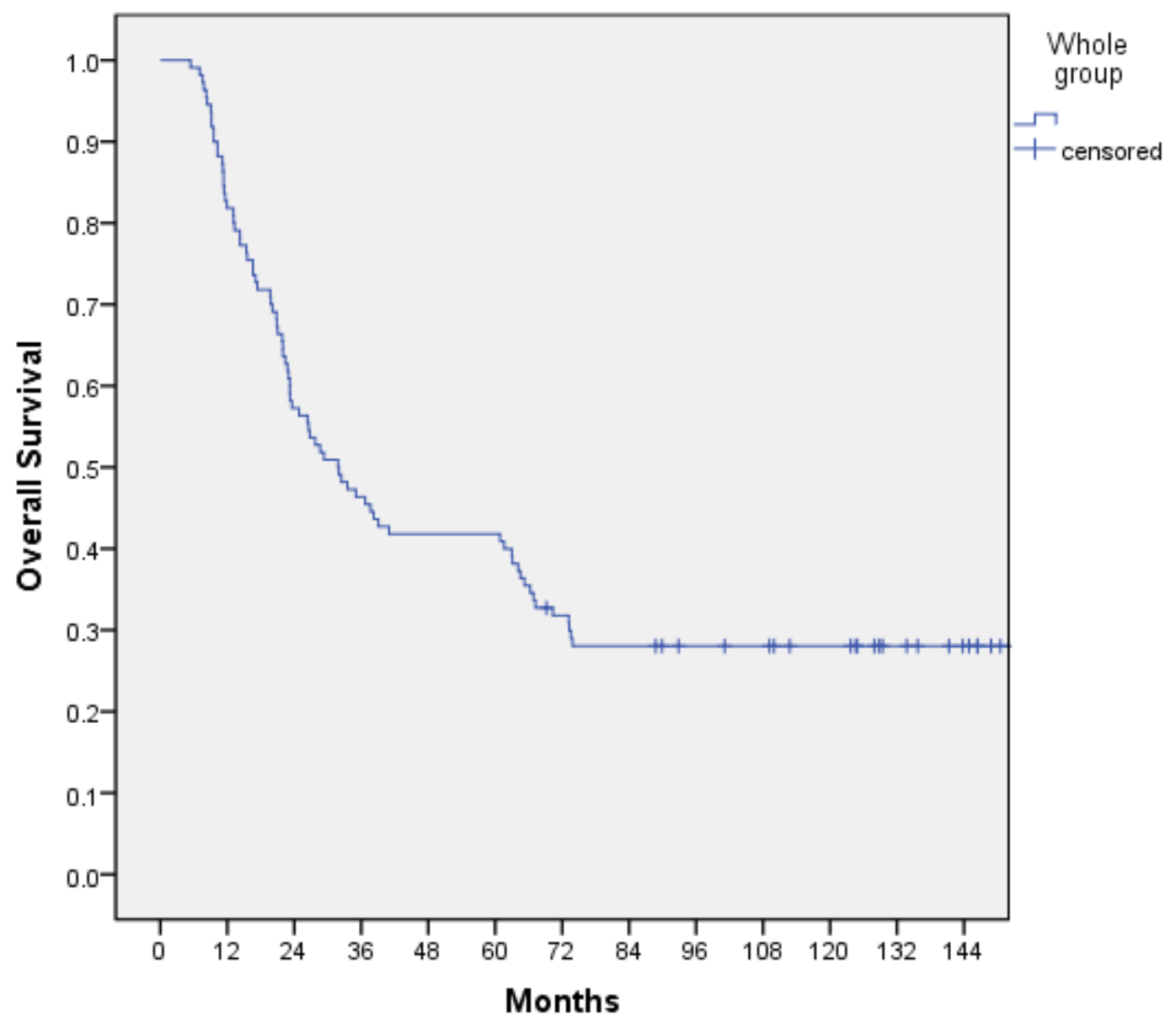

Figure 2

Survival rate for the whole group. 

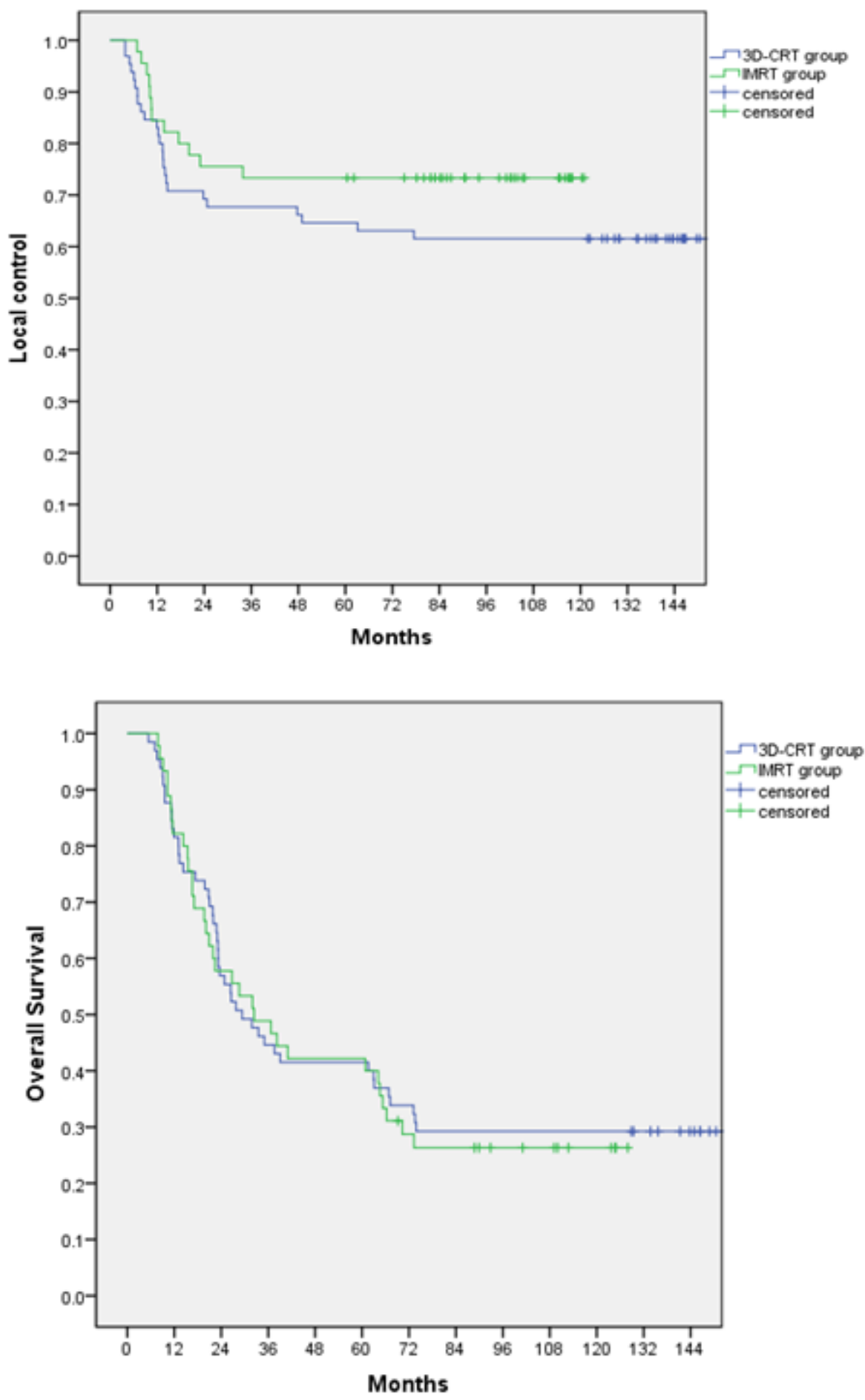

Figure 3

Local control and survival rates for the 3D-CRT and IMRT groups. 